\title{
Research Article \\ In-House versus In-Cloud ERP Systems: A Comparative Study
}

\author{
Ahmed Elragal and Malak El Kommos \\ German University in Cairo (GUC), New Cairo City, Egypt \\ Received 11 August 2012; Accepted 19 September 2012; Published 30 December 2012 \\ Academic Editor: Tey Lian Seng
}

\begin{abstract}
This paper provides a framework for comparison between the in-house implementation of ERP systems versus the in-cloud implementations. The paper first establishes a framework for the comparison based on three factors: pre-live, i.e., the implementation methodologies of both options; post-live, i.e., cost, time and the user-friendliness of the systems; and other factors, i.e., security and scalability. Results show that in-cloud systems are faster to implement, less costly and easier to use and scalable. In-house system, compared with in-cloud, gives organizations more control and hence many organizations deem them more secure.
\end{abstract}

Keywords: Enterprise Resource Planning (ERP) systems, Cloud Computing, ECC 6.0, ByDesign, comparative study.

\section{Introduction}

Nowadays, companies aim to achieve a competitive advantage among their competitors and gain customer satisfaction. This goal is achieved through the adoption of the latest trends of information technology (IT) (Gonçalves et al., 2011). In addition, Elragal and Al-Serafi (2011) stated that many companies have started to use enterprise resource planning (ERP) systems with the goal of improving business performance. Armbrust et al. (2010) stated that these ERP systems can be provided in an easier and more attractive way by their provision over the cloud which is still evolving and has the ability to make the IT industry more appealing to many users.

Cloud-based ERP systems are a point of attraction for companies that aim to achieve reduction of costs. Therefore, this paper will develop a framework for the comparison between the two systems. Then, the framework will be applied to SAP offerings, the in-house ECC 6.0 and the in-cloud SAP ByDesign.

The benefits of in-house ERP systems have been analyzed several times before; however, the focus on the benefits of in-cloud ERP systems has not yet been analyzed

Copyright (C) 2012 Ahmed Elragal and Malak El Kommos. This is an open access article distributed under the Creative Commons Attribution License unported 3.0, which permits unrestricted use, distribution, and reproduction in any medium, provided that original work is properly cited. Contact author: Ahmed Elragal E-mail: ahmed.elragal@guc.edu.eg

How to Cite this Article: Ahmed Elragal and Malak El Kommos, "In-House versus In-Cloud ERP Systems: A Comparative Study," Journal of Enterprise Resource Planning Studies, vol. 2012, Article ID 659957, 13 pages DOI: $10.5171 / 2012.659957$ 
thoroughly. There has not been a model provided for companies wishing to evaluate the benefits of using ERP systems over the cloud.

Trimi et al. (2005) stated that organizations adopting ERP systems face a lot of challenges, e.g., change management, costs related to cost of infrastructure, software licenses, consultant fees, in-house staff time, operating costs and user training costs.

The aim of this paper is to establish a framework for comparison between in-house versus in-cloud ERP systems and hence used by companies to make selection decisions. The importance of this topic lies within the widespread use of in-house ERP systems among companies, while the in-cloud ERP systems might be a better solution for some businesses like for example the case of small and medium sized enterprises (SMEs). This paper is trying to answer the following research question:

"What are the differences between implementing in-house ERP versus in-cloud systems with focus on benefits realizations? Benefits means user friendliness, less cost and less time".

\section{ERP Systems}

ERPs are most commonly defined as information systems that provide total integration of all key business activities, and automatically update new information into a single data repository accessible by all business functions, to allow coordination of all the business activities (manufacturing, purchasing, production planning, sales, accounting, etc) which add value to the business process operations (Abu Bakar and Zainol, 2008; Davenport, 1998; MozammelBin-Motalab and Shohag, 2011; Saini et al., 2011).

A survey results performed by Hawking et al. (2004) on 800 US-based companies shows that almost $50 \%$ of these companies have installed ERP systems and that these systems require $43 \%$ of the budget of the organization. Also, a report prepared by Computer Economics Inc. identifies that 76\% of manufacturers, $35 \%$ of insurance and health care companies and $24 \%$ of Federal Government agencies either have an ERP system installed or are considering such step.

However, Abu Bakar and Zainol (2008) presented that most ERP implementation projects or $90 \%$ of them are late or overbudget and that only 33\% are considered successful, which is obviously a very low rate. Davenport (1998) also stated that ERP systems implementation have many drawbacks that can affect a company's operation negatively because they enforce their own logic on a company's culture and operations, and their implementation requires many changes in the company.

In addition to that, Trimi et al. (2005) had to mention that the greatest challenge of ERPs is the costs related to these systems implementation which includes investing to acquire software, hardware, consultant fees, in-house staff (in charge of the installation process), staff operating on the system and finally the user training which is considered a very important cost influencing factor.

\section{Cloud Computing}

Cloud computing is defined as a new model where it is not necessary for users to physically own the resources such as hardware and software they need in their business, they can just use them over the internet. Such resources are offered, maintained and upgraded by third parties who own and manage them without any effort or involvement of the end users who just need to have minimal knowledge about how to access it, how to use it and how much it will cost. And this is the simple definition of cloud computing adopted by many authors, such as Armbrust et al. (2010), Hajjat et al. (2010), Mozammel-Bin-Motalab and Shohag (2011), Saini et al. (2011) and Senica et al. (2011). 
There are several types of clouds that make it flexible for adoption for all kinds of users. There are private clouds that are specified to a certain organization, community clouds shared by a community or a group of organizations, public clouds generally shared by the public and hybrid clouds which represent a mixture or combination of two or more types of clouds Armbrust et al. (2010) and Senica et al. (2011). This is explained in Figure 1 which represents the main types of clouds.

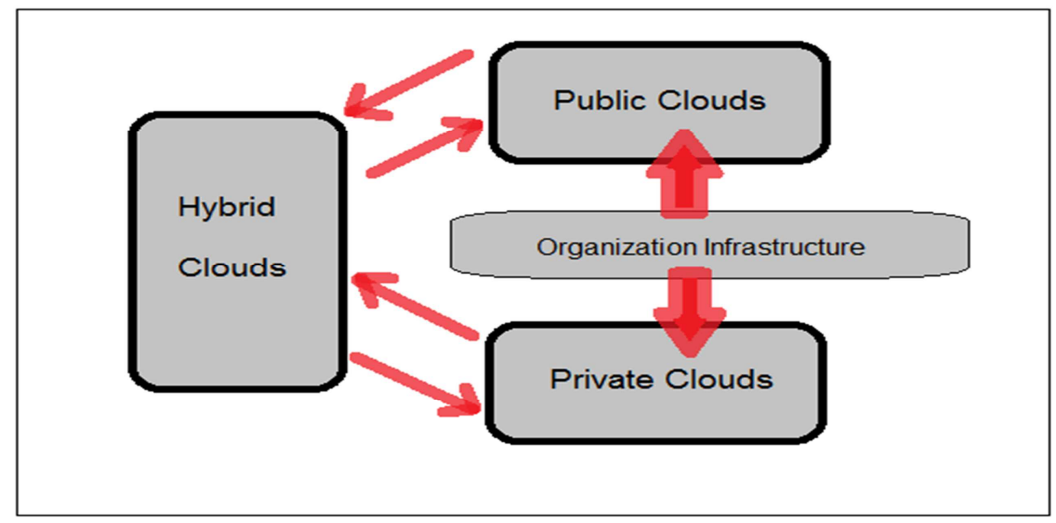

Figure 1. Types of Cloud Computing

It is widely believed by authors that the services offered by cloud computing can be divided into layers representing the types of services including the Software as a Service (SaaS), the Infrastructure as a Service (IaaS), and the Platform as a service (PaaS) (Saini et al., 2011) and Senica et al., 2011).

Also, concerning the benefits of cloud computing, they are becoming more obvious and cloud computing is starting to spread through many fields and is becoming more popular due to its utilization of the Internet. It provides users with the ability to pay as per their use of the resources provided on the cloud which was referred to by many authors as an "on-demand" solution (Senica et al., 2011) or "Utility computing" as per Mozammel-Bin-Motalab and Shohag (2011).

Using a cloud will result in lowering capital investments needed costs, yet services are provided in real-time. Furthermore, all the maintenance tasks have moved to the vendors including updating, upgrading, bugfixing the systems. In case of any problems, it is easy to recover without interruption of the business, while all information is shared, via the internet, by all business functions
(Armbrust et al., 2010; Hajjat et al., 2010; Kim et al., 2009; Saini et al., 2011).

However, security issues are the number one concern for companies planning the migration of their businesses to the cloud. Hackers may threaten confidential information that migrates into the cloud like for example in health care industry [9]. In addition, Armbrust et al. (2010) stated that, regarding the organizations that already have a large business and are considering the introduction of cloud computing in their businesses, these customers will be unwilling to migrate to the cloud environment without an analysis done in order to sustain the business continuity for cases and problems that could occur and affect the continuity of the business. Hajjat et al. (2010) analyzed that, the ambiguity of the applications performance on the cloud and organizations that may need to adopt several services from different providers may face a challenge during the integration process of these applications and data.

\section{Methodology}

For the purpose of this paper, and in order to explore the research question, a qualitative 
research approach was chosen seeking better and in-depth understanding and knowledge elucidation. A comparative case study would be the most suitable method to accomplish the research purpose using experiments as the research tool. Conducting an experimental research on two different systems both belonging to the company selected for the case study, which is SAP (Systems, Applications and Products in Data Processing). Whereby, ECC 6.0 represents the in-house system and Business ByDesign (ByD) representing the in-cloud system.

The comparison is broken-down into three factors: Pre-Live; Post-Live; and others. The methodology is shown in Figure 2. The PreLive stage is where a comparison between ASAP implementation methodology adopted for the in-house ERP systems and ByDesign Go-Live methodology adopted for the implementation of the in-cloud ERP systems. The comparison is based on the following factors: cost and time.

As for the Post-Live part of the analysis, a comparison was executed for the two systems in terms of time taken to perform certain processes i.e., user-friendliness. The final part of the comparison takes into consideration other factors influencing the choice of users between the two solutions. Those are security and scalability of both ByD and ECC 6.0.

Since the approach used is a case study research approach, generalizations could not be made since results are applied to cases under investigation. Figure 2 below explains the steps taken to accomplish the comparative case study.

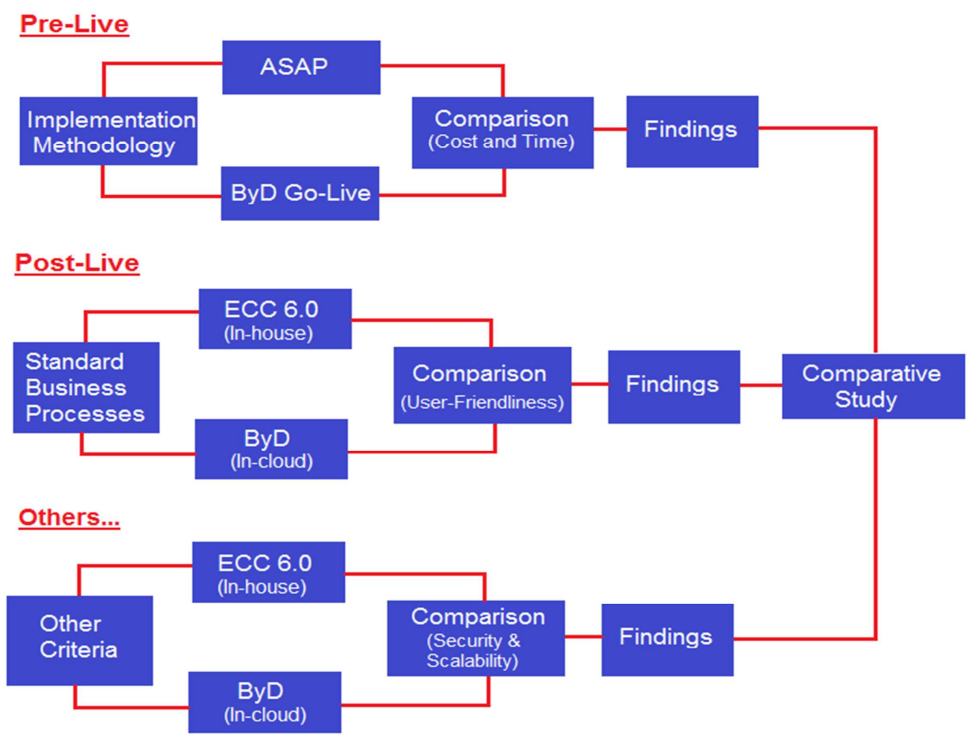

Figure 2. Methodology Diagram

\section{Comparative Case Study}

Since SAP is the major vendor of ERP systems with almost $50 \%$ of the market of companies using ERP systems according to Hawking et al. (2004), and Bögelsack et al. (2011) said that "SAP ERP systems represent the backbone of today's enterprises by supporting and automating nearly all business processes", the researchers thought it would be beneficial to perform a comparative study between two systems provided by SAP; one system representing the in-house solution and the other system representing the in-cloud solution.

In the following sub-sections, a comparative study will be explained between ECC 6.0 and 
ByD in terms of post-live factors, such as the user-friendliness of the system, and pre-live factors like cost and time associated with the implementation.

\section{Pre-Live (ASAP Implementation Methodology versus Byd Go-Live Methodology)}

Historically, implementing SAP systems has been looked at by many organizations as a major undertaking. Therefore, implementing a solid and tested implementation methodology is supposed to reduce the risk of implementation projects.

Therefore, SAP developed a simple implementation methodology to guide users through the implementation process which is called ASAP (Accelerated SAP), describing an implementation roadmap from a project management perspective to help direct the project team to optimize time, people and all resources required for the implementation in an efficient and comprehensive way. The ASAP implementation methodology as shown in Fig. 3 consists of five sequential phases starting with the preparation, blue printing, realization, testing \& deployment and finally the support.

Project Preparation is the first phase. There are also a number of tasks that need to be done to complete this phase, including: obtaining senior-level management and stakeholders' full agreement as they are the most important decision-makers of the company, identifying clear project objectives, architecting an efficient decision making process, creating an environment suitable for change and re-engineering by making sure that everyone in the organization is willing to accept the changes that will occur along with new SAP software, and finally building a qualified project team.

The second phase of the ASAP methodology is the Business Blueprinting. This is where the extraction of information related to the business takes place. The blueprints are in the form of questionnaires that are designed to investigate for information that would help discover how the company runs the business.

The third phase is the Realization, which is divided into two parts to configure the SAP system. First, the Baseline configuration based on the information collected in the blueprint document and second the Finetuning configuration that tackles approximately $20 \%$ of the configuration that was not handled in the Baseline configuration process. And the step following the configuration is to validate business processes, conduct organizational change management (OCM) and plan end user training.

The fourth phase is the Final Preparation, which consists of SAP training programs and severe functional and stress testing, including workload testing and integration or functional testing. This phase also concentrates on the fine-tuning of the configuration done previously before the GoLive phase and the migration of the data from the old system to the newly implemented SAP system. At the end of this phase, there is planning and documentation of a Go-live strategy. Preparation for Go-live means preparing for your end-users questions as they start actively working on the new SAP system.

The fifth and last phase of the implementation is the Go-Live and Support phase where everyone starts working on the new system. In order to achieve smooth GoLive without facing unwanted events, there should be a carefully done preparation process including consideration to what-if scenarios associated with business processes and the functioning of the technology supporting these business processes. And finally, also regarding the preparation, there should be a preparation for ongoing support, including essential maintenance contracts and documented processes and procedures. 


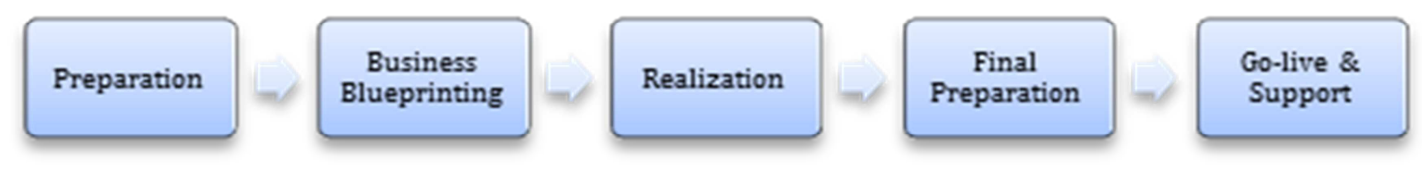

Figure 3. ASAP Implementation Methodology Phases

When SAP launched its cloud solution ByD, it had to tailor another implementation methodology best suitable for the new system. So the ByD Go-Live methodology was created to help the ByD users run their ERP projects in an efficient manner with the help of project phases, acceptance checkpoints, streams and project tasks. This methodology describes the tasks that have to be performed during the implementation projects and the sequence in which they should be executed. The ByDesign Go-Live Methodology shown in Figure 4 consists of only four phases starting with the preparation phase, followed by the fine-tune and integrate \& extend, then comes the test, and finally the go-live.

Phase one is the Prepare phase, which is a critical phase because it places the groundwork of the project and allows successful cooperation, creating a common understanding of the project scope and goals and how to achieve them. The key activities in this phase are: the project initiation, kick off, creation of the project schedule, review of the business scenarios and key business and process-related decisions. Furthermore, there is a study of relevant e-learning initiatives done by key users of the system. In the end, an infrastructure check is performed, followed by data cleansing, extracting and mapping activities. OCM is also introduced in this phase.

Phase two is the Fine-tune and Integrate \& Extend phase, where the two aspects are performed in parallel with each other. The fine-tune aspect focuses on the systemrelated activities, the establishment of organization structure and the establishment of all the system settings. Meanwhile, the integrate \& extend aspect of this phase focuses on the custom content development specified by the user and the data migration tasks. One of the most important activities in this phase is the data migration that includes maintaining basic data, testing data migration, migrating master data, migrating transaction data and validating data quality and integrity.

Phase three is the Test phase, which includes testing the end-to-end business scenarios in the system, loading the data and using it in testing the scenarios, and consequently any system or data issues are resolved until all test scenarios pass. The test phase is performed to ensure that all business scenarios and customer-specific business processes can be executed in the SAP ByD system with the migrated data. Once all the test scenarios have been run successfully or acceptable solutions have been identified for the test steps that failed, the Go-Live Readiness Acceptance Checkpoint is conducted to proceed with the following phase.

The fourth and final phase of the ByD Go-Live methodology is the Go-Live phase that focuses on the cutover tasks and the business and people readiness before the SAP ByD system is released to the end user for productive use. The activities of this final phase include the migration of all the data into the productive system and issuing internal and external communication. A final checkpoint is done to ensure that both the business and the people are ready for the new system and business processes. At the end of this phase the SAP ByD system will be live. 


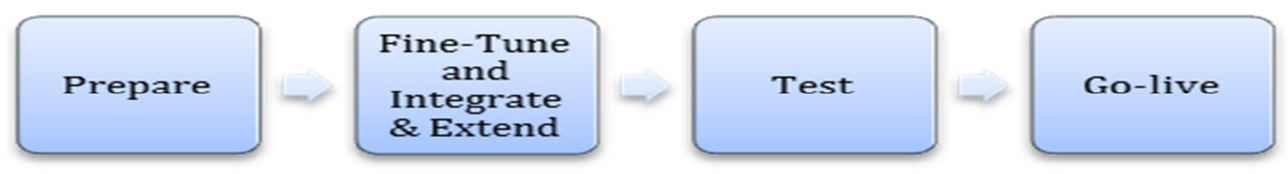

Figure 4. Byd Go-Live Methodology Phases

From the previous two figures and discussions representing the two methodologies from SAP, it is clear that the methodologies are different in terms of phases and activities, and their sequences as well. Further, the difference between the two methodologies is also possible considering the factors of time and cost. Below is an explanation.

\section{a) Cost}

The analysis of the costs associated with these implementations is highly important for companies because usually ERP implementation projects are highly costly and normally there are costs overruns. There are several cost-drivers related to ERP system implementation. These drivers include licensing, hardware, software, training, human resources (HR), data collection, data migration, etc.

Comparing the two systems, firstly, the licensing fees of ByD are far less than the inhouse ERP system given the following figures. The ByD monthly subscription costs $€ 30$ (€ 360 per year), while the yearly licensing fees of in-house ERP are $\$ 4,500(\approx$ $€ 3,500)$. The difference of costs is remarkable and acquiring ByD will offer a more economical solution.

Secondly, the hardware costs associated with ByD implementation will be far less due to several reasons. Less, or sometimes no, servers are needed compared to the in-house systems, accordingly, there are less administration duties requiring lower number of IT staff and hence much lower HR costs. In addition, the running costs that these servers need, like the cost of electricity and maintenance will be greatly minimized or even eliminated from the total cost burden of the company.

Regarding the software costs, the implementation of an in-house ERP system will necessitate investing high amounts of money for the server's software like the operating system (OS) and the data base management system (DBMS). These costs are already saved in the ByD implementations. Also, since ByD requires fewer, or no, machines to be installed, this entails saving the cost associated with their software acquisition as well.

The business scenarios collection is also more costly in the ASAP methodology (in the Business Blueprint phase), needing an "ASAP Question and Answer Database" in addition to an "Issues Database and Its Successor" which is a more redundant solution when compared to the ByD methodology where the business scenarios are already stored on the system and there is no need for additional expenses on tools needed for data collection and storage.

As for the comparison of the infrastructure of both systems, in the ASAP methodology there are three types of systems used to ensure a successful infrastructure setup for the project implementation; the Development (DEV) system, the Quality Assurance (QA) systems, the Production (PROD) system. As for ByD implementation, there are only checks performed to the company's existing infrastructure while eliminating the cost of these systems setup, in addition to the cost of the OS and machines accompanied by these systems; and therefore ByD again provides a more cost saving framework reducing redundancy to the maximum amount possible. 
Further, the costs needed to hire consultants and IT staff that will be responsible to monitor and maintain in-house ERP systems and handle the issues related to these systems would also be minimized and these responsibilities are transferred to the incloud system provider.

In summary, the in-cloud ByD systems will provide a low total cost of ownership by cutting off many costs needed for the infrastructure of ERP systems, the hardware acquisition, the software implementation costs, the maintenance costs and the updating costs, and provide a pay- as-you go model where companies are only required to pay a monthly subscription for the online use of the systems.

\section{b) Time}

Time is an important factor that influences the implementation process of ERP systems because usually most projects fail to meet expected due dates as contracted. Implementing $\mathrm{ByD}$, compared with regular in-house ERP implementation, would require less time due to several reasons that will be revealed in this section.

First, the time needed to hire HR to form the team who would be responsible of monitoring the servers and infrastructure would be less, if not eliminated, because the ByD implementation would not probably need servers and therefore would not need HR to administer it.

Concerning the business scenarios, in the ASAP methodology, there is the Business Blueprint (which is the second phase) designed to help extract relevant information about the business that is needed for implementation in addition to outlining the future business processes and business requirements. However, when it comes to the ByD methodology, the business scenarios are already stored on the ByD and ready to be reviewed by users during the first phase of the implementation process.
As to data migration process, where the company's data is being transported to SAP systems, ByD methodology has proven to be a more time-saving solution when compared to ASAP methodology. For ByD methodology, data is migrated to the new system from the beginning of the project during the first phase (Project Preparation phase). This enables the users to practice and test their new system and use their data on it from the beginning of the project, which means that the user is involved in the decision making process while having a good amount of knowledge about the system being implemented from the start.

The case is different when it comes to ASAP methodology as the data migration takes place later in the project in the fourth phase (the Final Preparation phase) right before the Go-Live phase.

Accordingly, the ByD methodology saves a lot of time required to perform the tests necessary to ensure that the system meets user expectations and organizational fit in addition to the elimination of the risks that may occur incase users have concerns regarding the system or need modifications to the system.

As for the configuration of an SAP system in ASAP methodology, it is divided into two parts; the Baseline configuration and the fine-tuning configuration. The baseline configuration is based on the information collected in the blueprinting phase and is followed by the fine-tuning phase tackling the exceptions that are not covered in the baseline configuration. When comparing this to the ByD methodology, it was found that implementing the system would only require a fine-tuning configuration dealing solely with the exceptions missed.

Moreover, a remarkable factor of comparison influencing the time spent on the implementation of ERP systems is the training time. ECC 6.0 requires a big amount of time training the employees who would be 
asked to perform their business work on the in-house ERP systems, and the ones who would be monitoring and maintaining these systems from an IT perspective after the GoLive. Whereas with the ByD solution, users are enabled on the system from the first phase of implementation and they can use their migrated data on the system from the very beginning; as for the time spent on training employees responsible for the maintenance and monitoring part, this time would be totally removed as these tasks are purely the responsibility of ByD providers who are in charge of making the customers live easier by handling the maintenance on their behalf.

In summary, the ByD minimizes the time needed to deploy and implement ERP systems and the ByD Go-Live methodology is a more agile framework allowing companies to save time spent on the implementation and infrastructure and focus on their business itself.

\section{Post-Live (User-Friendliness of ECC 6.0 versus ByD)}

Table 1 is presented to provide a comparison between the time taken by users of both ByDesign and ECC 6.0 to perform selected tasks. The tasks are: create customer master, create material master, create sales order, and search. Those four functions were selected arbitrarily for testing purposes.

The results show that the "Create Customer Master Data" business task performed on ByDesign system consumes (4:40 minutes) of the user's time, while performing this same business task on ECC 6.0 will take $(7: 10$ minutes) to be completed. Also, the "Create New Material Master" task on ByDesign takes (10:00 minutes) while it takes (12:40 minutes) to be performed on ECC 6.0. The third business process chosen for this comparison is the "Create Sales Order" task which takes (2:00 minutes) on ByDesign and (5:20 minutes) on ECC 6.0.

The last and most important, notable task is the "Search Function" which exists in both systems with the same concept. However, an ECC 6.0 user would have to spend $(2: 10$ minutes) waiting for the search function to find the required results, while a ByDesign user would only wait for a couple of seconds. And this is of course a significant factor that has caused ECC 6.0 to be slower than ByDesign.

Some may perceive that the difference, which is $(2: 30$ minutes, $2: 40$ minutes, $3: 20$ minutes, and 2:05 minutes) respectively, is not remarkable to a shocking extent that would push some businesses to replace ECC 6.0 by the ByDesign solution. However, if we are to analyze these timings on the long run and on many business processes performed by users every day, we would find that a regular ECC 6.0 user would be subject to spending hours each day waiting for the system to respond. For example, if a user performs 30 business processes a day using his search function, which consumes around 2 minutes per task, this means that this user will spend around one hour of their work day waiting for the search function to find some required text. 
Table 1. Comparison between ByD and ECC 6.0 in Terms of Speed of the System

\begin{tabular}{|l|l|}
\hline ByDesign & ECC 6.0 \\
\hline Create New Customer Master Data \\
\hline 4:40 minutes & $7: 10$ minutes \\
\hline Create New Material Master \\
\hline 10:00 minutes & $12: 40$ minutes \\
\hline Create Sales Order & $5: 20$ minutes \\
\hline 2:00 minutes \\
\hline Search Function & $2: 10$ minutes \\
\hline 5 seconds
\end{tabular}

\section{Other Factors}

Concerning the security of the systems, ByD is an in-cloud solution offered over the Internet, which means that the data will have to be stored and transported over the Internet. Therefore, the security of the data represents an issue regarding the adoption of ByD, as data will be transferred over networks and that makes vulnerabilities more common than the in-house system. As for the in-house system ECC 6.0, it is considered a more secure solution, as the data is safe against hackers as it is stored, locked and maintained on the company's premises.

The other factor influencing the comparison between ByD and ECC 6.0 is the scalability. It was analyzed that SAP's in-cloud solution (ByD) provides users with a more scalable solution where it can be flexible and can expand according to the user's demand and monthly or yearly subscription which allows the business to grow without worries about having to replace and upgrade the old system with a newer version that can be more suitable according to the different size of business demand. On the other hand, the ECC 6.0 system requires the user to handle such changes and upgrades relying on the internal team of consultants as well as budgets.

The following table provides a comparison between the ByD and ECC 6.0 according to selected criteria or factors to evaluate the user friendliness of both systems in comparison with each other and provide readers with a profitable framework for evaluation.

From the following table, it is seen that the cost and time taken to implement ByD is less than those taken for the ECC 6.0 implementation. Therefore, ByD is a more economic and time-saving solution. As for the user-friendliness, ByD system has a more friendly, transparent and interactive user interface, whereas the ECC 6.0 system is complex and its interface is rigid and hard to navigate which increases the time taken by the system's users to perform certain tasks and business processes and causes their disappointment regarding the performance of the system. 
Table 2. Comparison between Byd and ECC 6.0 in Terms of User-Friendliness and Others

\begin{tabular}{|l|l|l|}
\hline $\begin{array}{l}\text { Cost (to } \\
\text { implement) }\end{array}$ & Less & ECC 6.0 \\
\hline $\begin{array}{l}\text { Time (to } \\
\text { implement) }\end{array}$ & Less & More \\
\hline $\begin{array}{l}\text { User } \\
\text { friendliness } \\
\text { (process } \\
\text { time) }\end{array}$ & More & Less \\
\hline Security & Less & More \\
\hline Scalability & More & Less \\
\hline
\end{tabular}

\section{Conclusion}

Cloud computing is evolving dramatically and is expanding to include all services that can be provided online till it reaches the ability to include services for companies such as ERP systems provided over the cloud. The paper starts with providing an overview about ERP systems followed by an overview about cloud computing and the obstacles that these services have eliminated, making business more easier.

Most companies do not have the strength or will that motivates them to migrate their systems to the cloud, this is due to their low level of analysis and knowledge about the topic; for this reason a comparative case study identifying the benefits of using the incloud ERP systems was conducted. The comparative case study indicates that the incloud ERP systems have an effect on several aspects of a company, e.g., cost and time savings. In addition, the in-cloud system is more user-friendly and interactive, encouraging users or employees to work more efficiently than the ones working on the in-house system.
To conclude, ByD is most suitable for companies who have a high level of cost and time sensitivity, which means that the cost and time needed to implement in-house ERPs are higher. ByD is the appropriate solution for companies which do not have the capability to provide their system with large IT backbone required for the implementation and installation of these systems and which do not have the capability to hire and train the large number of employees who would be asked to perform their business work on these systems and the ones who would be responsible for maintaining and monitoring them.

SMEs are a very good example of the use of ByD. This is due to the relatively low number of employees and the fact that most of them cannot afford to spend the huge amount of time and money spent on the acquisition, implementation and maintenance of the inhouse systems. As for the large enterprises, which can afford the money and time required for in-house ERP systems, probably they will still opt for in-house systems to provide the security they need for their business. 


\section{Future Work}

In the future, more longitudinal case studies could be conducted to further investigate the difference between implementing in-house ERP versus in-cloud with focus on benefits realizations. The longitudinal study could also be useful in determining the Return on Investment (ROI) for both in-house and incloud systems and in providing a comparison between both in terms of speed of impacting the business in a profitable way; this could be achieved by performing quantitative researches to provide numerical data that would be useful in such analysis.

Furthermore, analyzing the different factors that would influence the quality of the systems in the post-live implementation phase should perform a comparison between the two systems in terms of quality. This would be a valuable study to be added to the time and cost factors analyzed in this paper. In addition, there should be studies analyzing the difference between user acceptance of both systems and the impact of culture over the adoption of both the in-house and incloud ERP systems. Finally, an industryspecific comparison can also be done to provide customers and vendors with a framework to base their decision according to their type of business.

\section{References}

Armbrust, M., Fox, A., Griffith, R., Joseph, A. D., Katz, R., Konwinski, A., Lee, G., Patterson, D., Rabkin, A., Stoica, I. \& Zaharia, M. (2010). "A View of Cloud Computing," In: Communications of the ACM, Vol. 53, No. 4, Pp. 50-58.

Bögelsack, A., Homann, M., Wittges, H. \& Krcmar, H. (2011). "Performance of SAP ERP with Memory Virtualization Using IBM Active Memory Expansion as an Example," In: Proceedings of the 5th International Workshop on Virtualization Technologies in Distributed Computing, San Jose, California, USA, Pp. 21-28.
Davenport, T. H. (1998). "Putting the Enterprise into the Enterprise System," In: Harvard Business Review, Vol.76, No. 4, Pp. 121-131.

Elragal, A. A. \& Al-Serafi, A. M. (2011). "The Effect of ERP System Implementation on Business Performance: An Exploratory CaseStudy," Communications of the IBIMA.

Gonçalves, A., Serra, N., Serra, J. \& Sousa, P. (2011). How to Use Information Technology Effectively to Achieve Business Objectives. In: Cruz-Cunha, M. M., and Varajao, J. (Eds.), Enterprise Information Systems Design, Implementation and Management: Organizational Applications. IGI Global: Hershey, Pennsylvania, United States, Pp. 2137.

Hajjat, M., Sun, X., Sung, Y. W. E., Maltz, D., Rao, S., Sripanidkulchai, K. \& Tawarmalani, M. (2010). "Cloudward Bound: Planning for Beneficial Migration of Enterprise Applications to the Cloud," In: SIGCOMM, New Delhi, India, Pp. 243-354.

Hawking, P., Stein, A. \& Foster, S. (2004). "Revisiting ERP Systems: Benefit Realization,"In: Proceedings of the 37th Hawaii International Conference on System Sciences. Island of Maui, Hawaii, USA.

Kim, W., Kim, S. D., Lee, E., Lee, S. \& Lee, S. (2009). "Adoption Issues for Cloud Computing," In: Proceedings of the 11th International Conference on Information Integration and Web-Based Applications \& Services, Pp.2-5.

Mozammel-Bin-Motalab, \& Shohag, S. A. M. (2011). 'Cloud Computing and the Business Consequences of ERP Use,' In: International Journal of Computer Application, Vol. 28, No. 8, Pp. 31-37.

Saini, S. L., Saini, D. K., Yousif, J. H. \& Khandage, S. V. (2011). Cloud Computing and Enterprise Resource Planning Systems. In: Proceedings of the World Congress on Engineering. London, UK. 
13 Journal of Enterprise Resource Planning Studies

Senica, N., Teixeira, C. \& Pinto, J. S. (2011). "Cloud Computing: A Platform of Services for Services," In: Cruz-Cunha, M.M., Varajao, J., Powell, P., Martinho, R. (Eds.), Enterprise Information Systems (Part 2) CCIS 220, Springer: Verlag Berlin Heidelberg, Pp.91-100.

Trimi, S., Lee, S. M., Olson, D. L. \& Erickson, J. (2005). "Alternative Means to Implement ERP: Internal and ASP," In: Industrial Management and Data Systems, Vol. 105, No. 2, Pp. 184-192.

Zaitun, A. B. \& Zaini, Z. (2008). "A Web-Based DSS for the Evaluation of an ERP System," Proceedings of the 10th International Conference of Information Integration and Web-Based Applications and Services. Linz, Austria: ACM Library, Pp. 698-701. 